\title{
«Le roman vit selon ses propres lois », ou comment les romanciers font du roman « le genre le plus libre qui soit »
}

"Fiction follows its own laws" or how novelists make the novel "the freest genre in existence"

\section{Jolianne Gaudreault-Bourgeois}

\section{OpenEdition Journals}

Édition électronique

URL : http://journals.openedition.org/tangence/624

ISSN : 1710-0305

Éditeur :

Université du Québec à Rimouski, Université du Québec à Trois-Rivières

Édition imprimée

Date de publication : 1 décembre 2018

Pagination : 103-118

ISSN : 1189-4563

Référence électronique

Jolianne Gaudreault-Bourgeois, « «Le roman vit selon ses propres lois », ou comment les romanciers font du roman « le genre le plus libre qui soit » », Tangence [En ligne], 118 | 2018, mis en ligne le 01 décembre 2019, consulté le 01 décembre 2019. URL : http://journals.openedition.org/tangence/624

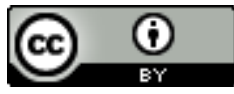

La revue Tangence est mise à disposition selon les termes de la Licence Creative Commons Attribution 4.0 International. 


\section{«Le roman vit selon ses propres lois», ou comment les romanciers font du roman «le genre le plus libre qui soit» Jolianne Gaudreault-Bourgeois Université McGill}

Le genre romanesque pourrait tout accueillir, tout englober. Tout pourrait y entrer et s'y amalgamer, comme dans la boutique du vieil antiquaire où pénètre Raphaël de Valentin dans La peau de chagrin, pièce encombrée où cohabitent des objets hétéroclites en provenance de tous les azimuts: peaux de serpent, brocantes, objets anciens, trésors perses et égyptiens. Telle est l'image forte que propose Jacques Rancière pour décrire le genre du roman dans son essai La parole muette. Dans cet ouvrage qui porte, selon ses mots, «sur les contradictions de la littérature», le critique fait du roman un genre sans genre, ou, plus précisément, le "genre de ce qui est sans genre» en ce que ce genre se voit dépourvu d'une «nature fictionnelle déterminée $»^{1}$.

Cette indétermination du roman est également relevée par de nombreux critiques, dont Nathalie Piegay-Gros qui en fait «la spécificité du genre ${ }^{2} »$. Ces critiques envisagent cette indétermination tantôt sous son angle thématique - le roman peut tout aborder tantôt sous son angle formel, le roman étant le genre ouvert se distinguant des autres genres, notamment du théâtre et de la poésie qui sont, eux, codifiés et soumis aux «règles». Mais ce sont surtout les romanciers eux-mêmes qui, dans le discours qu'ils tiennent sur le

1. Jacques Rancière, La parole muette, Paris, Hachette, coll. «Pluriel», 2011, p. 29.

2. Nathalie Piegay-Gros, "Introduction», dans Le roman, Paris, Flammarion, coll. «GF Corpus», p. 13. 
genre du roman, revendiquent cette indétermination, avec ceci de particulier qu'elle se présente pour eux sous le terme de «liberté». En effet, encore plus qu'un genre indéterminé, le roman est pour eux un genre «libre» et plus exactement «le genre le plus libre qui soit ${ }^{3}$ », selon l'expression qu'emploie René Boylesve dans une chronique de 1912.

Si un romancier comme Boylesve fait de cette liberté une notion plus théorique que pratique tant il prescrit la seule voie psychologique et réaliste pour le genre, chez les romanciers de la seconde moitié du $\mathrm{xx}^{\mathrm{e}}$ siècle, qui souhaiteront rompre avec les conventions, la liberté devient un enjeu crucial, voire obsessif. Cette observation devient du moins très flagrante lorsque l'on considère, de manière plus précise, la famille de textes que forment les essais sur le roman écrits par des romanciers depuis L'ère $d u$ soupçon de Nathalie Sarraute (1956). Ce corpus, que nous nous proposons d'analyser, comprend L'invitation au mensonge de Gilles Barbedette (1989), Roman du roman de Jacques Laurent (1977), Le roman en liberté de Félicien Marceau (1978) et Les testaments trahis de Milan Kundera $(1993)^{4}$. Si l'on peut parler d'un motif «obsessif» de la liberté dans ces textes, c'est que le terme revient sans cesse de même que l'idée que le roman est le «genre libre» par excellence.

Tous ces textes partagent globalement une même logique: leur auteur attribue une liberté créatrice intrinsèque ou fondamentale au genre romanesque et la pose comme inhérente à son existence. Cette liberté commande au roman de se soustraire aux règles qu'on tente — souvent de l'extérieur - de lui imposer. Mais dans le concret de la pratique romanesque, il est évident qu'il en va autrement: il ne peut pas ne pas y avoir de règles. De fait, dans les essais sur le roman écrits par des romanciers, le discours sur la «liberté» du genre s'accompagne généralement d'un discours sur les « règles» — ou encore sur les «lois» -, d'abord pour affirmer qu'elles existent et que le roman les défie, et ensuite pour dire que devant cette indétermination absolue, le roman est créateur de ses propres règles, de ses propres lois.

L'essai étant un lieu plus extensif de réflexion que peuvent l'être les formes plus courtes de la préface, de la chronique ou de l'entretien, ce que nous pouvons appeler un imaginaire du genre

3. René Boylesve, Opinions sur le roman, Paris, Plon, 1929, p. 55.

4. Il s'agit là d'une sélection d'essais sur le roman tirée d'un corpus plus large. 
romanesque a, dans les essais étudiés ici, tout l'espace nécessaire pour se déployer. Cet imaginaire et ce déploiement passent largement par une personnification du roman, qui se voit conférer des intentions et des attributs «humains». Pour le dire très simplement, ces essais font du roman un personnage, doté à la fois de traits physiques et de traits de personnalité. C'est ainsi que le roman naît et meurt - il peut aussi agoniser ou encore renaître de ses cendres - et entre ces termes, qu'il se livre à des aventures, des combats, des explorations, de grandes découvertes. De cette propension anthropomorphe témoigne tout particulièrement le titre de l'essai de Félicien Marceau qui aurait pu être "Le roman et la liberté», ou encore "La liberté $d u$ roman", mais se lit plutôt comme Le roman en liberté, c'est-à-dire le roman comme auteur de sa propre histoire, au double sens du terme.

En effet, les essais sur le roman écrits par des romanciers ont également comme caractéristique commune de raconter l'histoire du genre romanesque et ce, en des termes éminemment narratifs. Ils racontent cette histoire même lorsqu'ils prétendent ne pas le faire. Ainsi s'ouvre Le roman en liberté: "Qu'on ne cherche pas ici une histoire du roman. Il s'agit simplement de réflexions que, peu à peu, je me suis faites, tantôt en lisant des romans, tantôt en en écrivant ${ }^{5}$ ». Pourtant, s'il est une chose que le romancier-essayiste ne cessera de faire tout au long de son texte, c'est bien d'écrire une histoire du roman. Ajoutons que ces essais, considérés ensemble, ne déploient pas une, mais des histoires du roman, c'est-à-dire une variété d'histoires possibles, avec des commencements et des jalons très variés. Inconciliables sur le plan du contenu, ces histoires du roman présentent cependant une forme de cohérence par la récurrence de certains traits formels, notamment le rôle qu'y joue le motif caractéristique de la liberté, que nous nous proposons d'étudier ici, dans ses implications et ses paradoxes.

\section{Le roman contre la critique littéraire: la liberté contre les règles} (Laurent)

Le terme de «liberté», dans son acception négative, renvoie d'abord à l'absence de contraintes — ou encore de «règles", de «lois», si l'on souhaite utiliser les termes des romanciers étudiés.

5. Félicien Marceau, Le roman en liberté, Paris, Gallimard, coll. «NRF», 1978, p. 7. Désormais, les références à cet ouvrage seront indiquées par le sigle $R L$, suivi de la page, et placées entre parenthèses dans le corps du texte. 
Ainsi, à l'intérieur de l'histoire du roman qu'ils écrivent, un des principaux défis du roman est son face-à-face avec les «règles» de toutes sortes. Mais quelles sont-elles? Sous-entendues plus qu'identifiées, il s'agirait, suivant les romanciers, de celles de la bienséance, de la vraisemblance, de la morale, de la composition.

De tous les essais, Roman du roman de Jacques Laurent est sans doute celui qui insiste le plus sur ces relations d'opposition en construisant une histoire du roman marquée, pour ne pas dire scandée, par de nombreuses luttes qui opposent le genre romanesque à des «règles». Ainsi, si «Laclos est frappé par le pouvoir du roman d'échapper aux règles de la bienséance» et si à cette époque le roman est «libre d'être libre», arrive l'heure où " une caste s'apprête à faire entrer le roman dans le rang», écrit-il, car, pour elle, « un genre a des règles ${ }^{6}$. C'est la caste des professeurs, principaux antagonistes $\mathrm{du}$ roman chez Jacques Laurent, car Brunetière, Villemain, La Harpe, Taine, Sainte-Beuve et quelques autres auraient entrepris de soumettre le roman à une "juridiction» $(R R, \mathrm{p} .153-154)$. Laurent campe d'un côté le genre du roman, de l'autre la «vieille garde» de la critique littéraire et cet affrontement a lieu sans surprise sous le signe de la naissance du roman. En effet, non seulement les romanciersessayistes font tous «naître» le roman à un moment précis, mais ce commencement se montre toujours comme une forme de combat déterminante pour la suite de son histoire ${ }^{7}$. Laurent écrit:

[I]l va de soi que les critiques ne peuvent pas renoncer à leurs règles de l'unité d'action et de l'unité psychologique puisque, sans règle, ils seraient sans pouvoir. Au long du xix ${ }^{\mathrm{e}}$ siècle, une critique universitaire que je hais comme Balzac la hait, s'est constamment appliquée à imposer les normes d'une prétendue esthétique à un genre qui était né de sa liberté et s'était nourri de son dérèglement. $(R R, \mathrm{p} .156)$

6. Jacques Laurent, Roman du roman, Paris, Gallimard, coll. «Nrf», 1977, p. 153154. Désormais, les références à cet ouvrage seront indiquées par le sigle $R R$, suivi de la page, et placées entre parenthèses dans le corps du texte.

7. Comme l'a montré Sophie Rabaud au sujet des récits de commencement du roman dans le discours critique. Voir Sophie Rabaud, «Il était plusieurs fois le roman ou comment les critiques narrent les commencements du roman », dans Jean Bessière (dir.), Commencements du roman, Paris, Honoré Champion, 2001, p. 49-63. 
Le roman ressort temporairement domestiqué de cet affrontement, alors que les romanciers se mettent eux-mêmes à produire des théories ou à soumettre le roman à des règles, comme Zola qui, avec son programme scientifique, "aurait risqué d'emprisonner définitivement le roman, donc de l'exterminer» $(R R, \mathrm{p} .160)$. Les démêlés du protagoniste-roman avec les «règles», elles aussi personnifiées, s'échelonnent sur plusieurs pages qui traversent les quelques décennies littéraires du tournant du siècle. Mais, alors que les professeurs et les critiques n'ont de force que celle des règles, le roman a pour lui la force de la vie et celle de sa liberté qui a permis, selon Laurent, cité plus haut, son auto-engendrement. La «liberté» atteint dès lors son acception positive: l'autonomie d'un sujet (rationnel), sa possibilité d'agir, alors que se présente l'issue du dernier duel, qui avait opposé cette fois le roman à Sainte-Beuve et Bourget. Contre cette critique littéraire, qualifiée de «système qui depuis l'art visait à discipliner le roman » et qui était toujours prête à le juger selon « des règles draconiennes» $(R R, \mathrm{p} .166)$, Jacques Laurent écrit:

Le roman triomphe en liberté, triomphe grâce à sa liberté, triomphe aussi grâce à son triomphe car celui-ci incite, par la diversité de ses fruits, les talents les plus divers à choisir ce mode jadis mineur et frivole pour s'exprimer. En un autre temps, Montherlant se serait peut-être limité au théâtre et Aragon, satisfait de sa poésie, n'aurait pas songé à atteindre les sommets de la prose romanesque. Le roman aimante toutes les inspirations parce qu'il n'impose ni même ne propose aucune forme. Il y a des parentés entre un roman de Stendhal, de Vigny, de Balzac, il n'y en a pas entre un roman de Proust et un roman de Drieu la Rochelle, il n'y en a pas entre Gatsby et L'Épithalame, Sanctuaire et Lewis et Irène, Moïra et Les jeunes Filles, entre Les enfants terribles et Les Hommes de bonne volonté, Autant en emporte le vent et L'irrésistible, Nadja et Le Noud de vipères, entre Amants heureux amants et La Nausée, entre Mademoiselle de la ferté et Notre-Damedes-Fleurs, entre Monsieur Godeau intime et Voyage au bout de la nuit, Une chambre à Manhattan et La conspiration, entre Regain et $42^{e}$ parallèle, entre Choix des élues et La condition humaine, entre le Voyage de Shakespeare et Les faux-Monnayeurs, entre La Montagne magique et Cyclone à la Jamaïque, entre Le Soleil se lève aussi et Les beaux Quartiers, entre L'Homme sans qualités et Le diable au corps. La littérature fond dans le roman. 
Ce fut l'âge du roman. Sa liberté lui donnait un pouvoir d'attraction qui déterminait beaucoup d'écrivains à préférer le mode d'expression romanesque à ceux vers lesquels ils inclinaient naturellement. (RR, p. 176-177)

Plusieurs éléments sont à noter ici. D’abord la stratégie rhétorique de l'énumération de titres qui rappelle l'argumentation de la préface à Pierre et Jean de Maupassant, romancier auquel Laurent fait d'ailleurs référence quelques pages plus tôt. Dans cette préface, où il

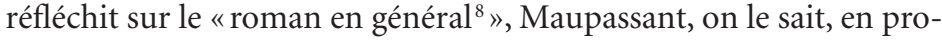
fite pour régler ses comptes avec la critique littéraire, trop encline à juger les romans selon des règles fixes:

Existe-t-il des règles pour faire un roman, en dehors desquelles une histoire écrite devrait porter un autre nom? Si Don Quichotte est un roman, Le Rouge et le Noir en est-il un autre? Si Montecristo est un roman, L'Assommoir en est-il un? Peut-on établir une comparaison entre Les Affinités électives de Goethe, Les Trois Mousquetaires de Dumas, Germinal de M. Zola? Laquelle de ces œuvres est un roman? Quelles sont ces fameuses règles? D'où viennent-elles? Qui les a établies? En vertu de quel principe, de quelle autorité et de quels raisonnements? Il semble que ces critiques savent d'une façon certaine, indubitable, ce qui constitue un roman et ce qui le distingue d'un autre qui n'en est pas un. [...] Un critique intelligent devrait, au contraire, rechercher tout ce qui ressemble le moins aux romans déjà faits, et pousser autant que possible les jeunes gens à tenter des voies nouvelles 9 .

C'est l'autorité, voire la légitimité de la critique littéraire qui se voit discréditée par le romancier dont l'argument principal est le fait même de la diversité des œuvres romanesques publiées et reconnues comme telles. Or, chez Laurent, il ne s'agit pas d'argumenter - il n'a pas à se battre contre Sainte-Beuve, même s'il se montre, on l'apprend plus loin, plus qu'agacé par la critique littéraire de son temps (la Nouvelle critique) —, mais avant tout de raconter, c'est-àdire, en l'occurrence, de raconter comment le roman triomphe grâce à sa toute puissante liberté, car dans tous les essais étudiés ici, le roman finit par l'emporter. Il s'agit donc de dire sa victoire, laquelle converge vers cet «âge du roman» qui coïncide non pas avec l'âge

8. Guy de Maupassant, Pierre et Jean [1887], Paris, Garnier et Flammarion, 1992, p. 16.

9. Guy de Maupassant, Pierre et Jean, ouvr. cité, p. 17. 
d'or du roman réaliste, mais plutôt avec le moment où le roman s'est arrogé le maximum de libertés formelles, le moment où le roman éclate sur le plan de la forme, soit le premier $\mathrm{xx}^{\mathrm{e}}$ siècle, si l'on en croit les titres énumérés. À cette époque, semble dire Laurent, jamais deux romans ne se ressemblent, tellement les possibilités formelles sont nombreuses, pour ne pas dire infinies.

L'âge du roman et sa «désinvolture»: la liberté de jouer (Marceau, Kundera)

Dans Roman du roman, la liberté est, d'une part, présentée comme la caractéristique « innée» du genre - il naît avec elle et par elle - mais, d'autre part, elle est associée à un certain moment de son histoire qui semble l'exprimer pleinement. Cette ambiguïté (ou ce double statut) apparaît chez d'autres romanciers-essayistes dans leur discours visant à faire de la liberté le grand trait définitoire du genre romanesque, notamment dans Le roman en liberté de Félicien Marceau et dans Les testaments trahis de Milan Kundera qui ont en commun de lier cet âge à une caractéristique particulière du roman: sa «désinvolture», indissociable de sa liberté.

$\mathrm{Si}$, dans Le roman en liberté, Félicien Marceau parle surtout du $\mathrm{XIX}^{\mathrm{e}}$ siècle et du roman réaliste, il associe néanmoins lui aussi plus spécifiquement la liberté au roman $\mathrm{du} \mathrm{xx}^{\mathrm{e}}$ siècle. Son propos est emblématique de la tension qui existe, dans le discours essayistique des romanciers, entre la liberté comme essence du roman et la liberté comme moment de son histoire. D'un côté, Marceau n'hésitera pas à faire lui aussi du roman - en utilisant exactement les mêmes mots que René Boylesve cité plus haut - «le genre le plus libre qui soit» ( $R L$, p. 94). Il ajoute, usant d'une métaphore spatiale: «C’est un royaume dont les frontières se confondent avec l'horizon» ( $R L, \mathrm{p} .94)$, figurant ainsi toute l'étendue et l'ouverture du genre, qu'il tente de définir sous plusieurs de ses facettes, et, dans le chapitre d'où est tirée cette citation, en fonction de son caractère «documentaire». Par-là, il pointe la propension des romanciers - surtout ceux du XIX ${ }^{\mathrm{e}}$ siècle pour le travail minutieux de collecte et de compilation d'informations diverses aux fins du roman en cours, sorte de règle du métier qui inscrit le genre de plain-pied dans la «réalité». Ainsi, se demande-t-il

[si] le roman ne doit pas cette liberté précisément à cette solidité, à cette solidification, à cette exactitude dont Balzac, Flaubert et Zola 
se sont tant préoccupés, si ce n'est pas grâce à eux que le roman se présente maintenant avec un préalable de crédibilité, si ce n'est pas parce que leurs personnages étaient si exactement décrits ou si opaques qu'il peut maintenant évoluer avec plus de désinvolture [...]. (RL, p. 94)

Ainsi, c'est parce que le roman, à un certain moment de son histoire - ou encore à un certain âge de sa vie, pouvons-nous presque lire — , s'est montré sérieux, s'est donné des règles, avec tout le travail de détails et d'exactitude que cet art suppose, qu'il peut maintenant se permettre d'être aussi libre, d'évoluer dans sa pleine «désinvolture», en quelque sorte, comme quelqu'un qui aurait travaillé très fort pendant les années importantes de sa vie, pour ensuite profiter d'une retraite libre, confortable et pleinement méritée.

Milan Kundera, dans Les testaments trahis, parle lui aussi de la «liberté désinvolte» du roman, non pas pour caractériser le «maintenant» du roman (ou plus largement le $\mathrm{xx}^{\mathrm{e}}$ siècle), mais plutôt en référence à un passé lointain. Au contraire de Marceau qui dit ne pas vouloir écrire une histoire du roman, Kundera se fait délibérément historien du genre et propose de scinder cette histoire en deux grandes «mi-temps». La première s'étendrait du début du XVI ${ }^{\mathrm{e}}$ siècle jusqu'à la fin du XvIII ${ }^{\mathrm{e}}$, de Rabelais à Sterne, en passant par Cervantès et Diderot, quatre figures qu'il affectionne particulièrement. Pour Kundera, cette première mi-temps correspond à l'époque où le roman est davantage «improvisation» que "composition ${ }^{10}$. Le roman était alors encore libre des règles qui vont par la suite s'imposer. À ce titre, il fait de Jacques le fataliste l'œuvre emblématique de cette période où la liberté romanesque foisonne et «se moque de la règle de l'unité d'action» (TT, p. 29), le roman ne se donnant pas encore la mission de faire concurrence à l'état civil, selon la célèbre formule de Balzac. Avec Balzac et Dostoïevski, romanciers de la deuxième mi-temps du roman, l'art de la composition s'impose, nous dit Kundera, car les intrigues complexes où se croisent

10. «La liberté par laquelle Rabelais, Cervantes, Diderot, Sterne nous envoûtent était liée à l'improvisation. L'art de la composition complexe et rigoureuse n'est devenu nécessité impérative que dans la première moitié de XIx ${ }^{\mathrm{e}}$ siècle» (Milan Kundera, Les testaments trahis [1993], Paris, Gallimard, coll. "Folio», 2000, p. 28). Désormais, les références à cet ouvrage seront indiquées par le sigle $T T$, suivi de la page, et placées entre parenthèses dans le corps du texte. 
les destinées de plusieurs personnages exigent un plan «minutieusement calculé des actions et des scènes» (TT, p. 28).

Face aux contraintes que rencontre le roman de la deuxième mi-temps - contraintes qui, chez Kundera, au contraire de chez Laurent relèvent davantage de son histoire que de faits externes le roman de la première mi-temps nous apparaît dans toute sa désinvolture, dans toute sa jeunesse, et comme étant doté d'une «liberté joyeuse» (TT, p. 12), où tout peut arriver, tout peut survenir, sans impératif de vraisemblance: «Le romancier de notre siècle, nostalgique de l'art des anciens maîtres du roman, ne peut renouer le fil là où il a été coupé; il ne peut sauter par-dessus l'immense expérience du XIX ${ }^{\mathrm{e}}$ siècle; s'il veut rejoindre la liberté désinvolte de Rabelais ou de Sterne, il doit la réconcilier avec les exigences de la composition.» (TT, p. 29)

Mais comment concilier construction et improvisation? Comment réinsuffler de la jeunesse, ou de la "liberté désinvolte», au genre romanesque, sans faire fi de son âge adulte, sans renier le principe de construction qui lui est devenu inhérent? C'est en quelque sorte le défi que pose l'histoire du roman au romancier du $\mathrm{xx}^{\mathrm{e}}$ siècle. La réponse de Milan Kundera à ce défi repose sur l'idée de jeu. Le romancier doit jouer, comme le font par exemple Kafka, Gombrowicz et Herman Broch qui ont «rendu plus libre la composition; reconquis le droit à la digression; insufflé au roman l'esprit du non-sérieux $[\ldots] »(T T$, p. 91$)$ - nous pouvons reconnaître là ses trois autres figures de prédilection — et qui traitent des sujets les plus sérieux dans un esprit ludique, en se souciant peu des règles de la vraisemblance.

Ainsi, le romancier doit, comme le fait Kafka, renoncer à certaines règles, «renoncer aux dogmes du réalisme psychologique» ( $T T$, p. 91) pour se recentrer vers des règles qu'il se crée lui-même:

[L]e jeu, qu'est-ce que c'est, en fait? Tout jeu est fondé sur des règles, et plus les règles sont sévères plus le jeu est jeu. Contrairement au joueur d'échecs, l'artiste invente ses règles luimême et pour lui-même, et plus les règles sont sévères plus le jeu est jeu; en improvisant sans règle, il n'est donc pas plus libre qu'en inventant son propre système de règles. (TT, p. 30)

Kundera s'étend peu sur la question et ne dit pas exactement en quoi consistent ces règles du jeu. Il se contente de les examiner dans 
deux romans qu'il cite en exemple: Les somnambules et Les versets sataniques qu'il juge construits selon des règles musicales et polyphoniques ${ }^{11}$. Plus généralement, les lois se rapportant à une «troisième mi-temps» de l'histoire du genre du roman sont celles qui concernent l'imagination, et, encore une fois, la «liberté» créatrice du roman. Kafka, par son traitement ludique du thème de l'organisation sociale, notamment avec L'Amérique et Le château, incarne là encore, selon Kundera, la voie à suivre pour le roman, celle d'une littérature qui a donné à l'imagination «toute la liberté nécessaire (liberté d'exagération, d'énormités, d'improbabilités, liberté d'invention ludique)»(TT, p. 100).

\section{Quand le romancier découvre la «formidable liberté» de son art (Barbedette, Malraux, Marceau, Laurent)}

L'«âge» associé à la liberté du roman peut aussi être celui que doit atteindre le romancier avant de pouvoir tirer profit des ressources du genre. C'est le cas dans L'invitation au mensonge de Gilles Barbedette. Au même titre que Milan Kundera, Barbedette souhaite sortir le roman de l'impératif de réalisme qui, soutient-il, continue de peser sur lui. Mais, alors que Kundera opte pour une sortie par le jeu, Barbedette préconise un recours à une certaine part de folie. L'invitation au mensonge incite le romancier à trahir le réel, à cesser de vouloir bêtement l'imiter. Le romancier doit, à l'instar de Nabokov, plonger au fond de lui pour y découvrir une étrangeté et une folie propres au roman:

Il y a eu un Homère, un Proust, un Tolstoï avant nous. Mais on ne les «remplacera» pas. On peut les imiter ou tenter de recommencer leur expérience, mais une fois abandonnée cette enfance nécessaire de l'imitation d'épigone, tout écrivain doit réaliser que l'apprentissage du roman passe par la domestication d'une

11. «Exemple: le troisième livre des Somnambules de Broch qui est un fleuve "polyphonique" composé en cinq "voix", cinq lignes entièrement indépendantes: ces lignes ne sont liées ensemble ni par une action commune ni par les mêmes personnages et ont chacune un caractère formel tout différent (A-roman, B-reportage, C-nouvelle, D-poésie, E-essai). Dans les quatre-vingt-huit chapitres du livre, ces cinq lignes alternent dans cet ordre étrange: $A-A-A-B-A-C-$ A-A-D-E-C-A -B-D-D-D-A-E-A-A-B-E-C-A-D-B-B-A-E - [... . Qu'est-ce qui a poussé Broch à choisir justement cet ordre et pas un autre? [...] Pas la logique des caractères ou de l'action $[\ldots] »(T T$, p. 30$)$. 
étrangeté à travers l'héritage romanesque qui lui a été légué. C’est cela la formidable liberté du roman ${ }^{12}$.

Tout se passe comme si la véritable phase de création et de liberté du romancier était précédée d'une étape préalable où il se fait la main en tentant d'imiter les grands maîtres. Mais l'histoire d'un art ayant horreur de la répétition - selon une idée que Barbedette emprunte à Kundera - , le romancier n'a d'autre choix que de cesser d'écrire en s'inclinant devant les monuments littéraires. Arrive l'heure où il doit se concentrer sur sa propre folie, tout en confrontant la liberté du roman et en tentant de l'apprivoiser.

Pour ce faire, le romancier doit d'abord rencontrer cette liberté, la découvrir. C'est là un autre lieu commun des essais sur le roman: la découverte, par le romancier, de sa liberté, même si celle-ci est d'abord celle de son art. Cette étape constitue souvent un épisode central de l'histoire du genre romanesque racontée par les romanciers. La découverte de la liberté du roman par les romanciers peut aussi se présenter comme celle d'une liberté morale de contenu. Dans Le roman en liberté, Marceau soutient, sans que l'on s'en étonne, qu'il est possible de tout exprimer dans un roman, celui-ci échappant plus facilement à la censure que les autres genres, notamment le théâtre. Marceau multiplie les exemples, en commençant par la pièce Vautrin, écrite par Balzac et interdite de représentation le lendemain de sa première (en 1840), alors que les romans où figurent le même Vautrin, et, dit-il, «avec bien plus de virulence» $(R L$, p. 70$)$, ne rencontrent jamais ce type de problèmes. Pour Marceau, c'est cette découverte - celle d'une liberté morale - qui aurait fait adhérer ceux que l'on considère comme les plus grands romanciers du XIx ${ }^{\mathrm{e}}$ siècle au genre du roman: " $[\mathrm{C}]$ hez Stendhal comme chez Balzac, l'adhésion au roman a été précédée ou accompagnée d'une réflexion sur le roman, d'une découverte de ses possibilités, d'une découverte de sa liberté. Liberté de mouvement, liberté de l'écriture, mais aussi liberté politique.» $(R L$, p. 70$)$

Dans L'homme précaire et la littérature d'André Malraux, que nous nous permettons de convoquer $\mathrm{ici}^{13}$, cette découverte est plus

12. Gilles Barbedette, L'invitation au mensonge. Essai sur le roman, Paris, Gallimard, coll. «NRF essais», 1989, p. 133.

13. Un texte qui, soulignons-le, ne fait pas directement partie du corpus, car il n'est pas à proprement parler un essai sur le roman, mais plutôt un essai sur la littérature où il est largement question du roman. André Malraux, L'homme précaire et 
précisément celle des possibilités formelles que le genre recèle et qui permettent au romancier de faire du roman autre chose que de «l'histoire racontée»:

[L]a fiction échappe aux règles qu'elle prétend s'imposer parce qu'on ne passe que par escamotage, du Grand Cyrus à La Nouvelle Héloïse, ou de La Nouvelle Héloïse au Père Goriot. Il ne s'agit pas de raconter d'autres histoires, de les raconter autrement; il s'agit de la découverte, par le romancier, de son ubiquité, de son omniscience, de sa liberté, de l'autonomie de ses œuvres qui ne se limitent plus aux histoires et aux contes. Peu à peu, il découvrira l'existence de ce qui, dans le roman, n'est pas l'histoire racontée. (HPL, p. 111)

Cette découverte permet au romancier de défier les (soi-disant) règles qui font du roman un simple récit, la liberté résidant pleinement, pour Malraux, dans la part du roman qui excède la logique de la diégèse. Ce faisant, cette découverte que fait le romancier d'une liberté dans la forme est aussi ce qui permet au genre de se transformer ou «d'évoluer».

Cette découverte, Jacques Laurent, dans Roman du roman, la met directement en scène à l'intérieur d'un récit anecdotique et autobiographique. Le romancier raconte avoir écrit son premier roman autour de 1942, soit au moment où Kleber Haedens rappelait que le roman n'était pas «un service public et qu'il n'obéissait à aucune règle», et que même Gide, malgré son vieil attachement à une certaine poétique de la forme, consentait à ce que «les règles n'existassent que dans l'esprit de quelques critiques attardés» $(R R$, p. 148). Il s'agit là du contexte historique de l'écriture de ce premier roman, mais plus intéressantes encore sont les conditions spatiales dans lesquelles s'effectue cette découverte chez Jacques Laurent:

[J]'avais démarré ce qui devait être mon premier roman, vivant au milieu des bois et bien étranger aux disputes littéraires. Or, je constate que la question ne se posait pas pour moi de savoir s'il était bon ou mauvais de respecter ou d'enfreindre les lois du roman. Il allait de soi que la liberté créative était son essence et l'avait toujours été $[\ldots]$. La liberté ne se manifestait pas pour

la littérature [1977], éd. Christiane Moatti, Paris, Gallimard, coll. «Folio Essais », 2014. Désormais, les références à cet ouvrage seront indiquées par le sigle HPL, suivi de la page, et placées entre parenthèses dans le corps du texte. 
moi comme une supériorité sur les arts soumis à des contraintes, mais comme une nécessité et, depuis le début du siècle, elle était si peu contestée qu'elle me semblait faire corps avec le roman. Or le roman était apparu grâce à un concours de circonstances qui lui avait permis d'être libre, c'est-à-dire d'être. $(R R, \mathrm{p} .149)$

Dès lors, la liberté du genre romanesque - et celle-ci, à nouveau, explique son commencement dans l'histoire - se voit associée à la liberté de la personne du romancier dans une fusion des instances narratives. En effet, le romancier effectue ici ce que les théories de l'ethos et de la posture désignent comme une "présentation de soi ${ }^{14}$ » ou une "mise en scène de l'auteur ${ }^{15}$ ", soit celle d'un écrivain qui vit dans les bois, loin des centres urbains où sévissent les disputes littéraires. À l'instar du «roman en liberté», pour reprendre le titre de l'essai de Marceau, nous avons donc affaire à un «romancier en liberté».

\section{L'imaginaire corporel d'un genre littéraire (Malraux, Marceau)}

Si la liberté peut aussi facilement «faire corps avec le roman", selon l'expression de Laurent, c'est peut-être parce que le roman, dans les essais qui portent sur lui, se voit doté d'un corps physique. Certes, un principe de «vie» est depuis des siècles rattaché à l'imaginaire des genres littéraires. C'est ce que Marielle Macé appelle le «paradigme biologique ${ }^{16}$ » de la critique générique, qu'il s'agisse de l'évolutionnisme de Brunetière ou de la conception organique de la tragédie qui marque déjà la Poétique aristotélicienne. Mais le fait que cette vie soit à ce point exacerbée dans les essais sur le roman produits par les romanciers est à mettre en lien avec le fait que le genre du roman n'a jamais été codifié, la métaphore biologique devenant alors la seule forme d'organisation disponible pour le définir. En effet, si le roman est «le genre le plus libre qui soit», il n'y règne pas pour autant une anarchie totale. Or, mise à part la piste du "jeu» suggéré par Kundera, pour la plupart des romanciers-essayistes, les seules lois aptes à le gouverner de manière légitime sont les lois

14. Voir Ruth Amossy, La présentation de soi. Ethos et identité verbale, Paris, Presses universitaires de France, 2010.

15. Voir Jérôme Meizoz, Postures littéraires. Mises en scène moderne de l’auteur, Genève, Slatkine, 2007.

16. Marielle Macé, «Introduction», dans Le genre littéraire, Paris, Flammarion, coll. «GF Corpus», 2004, p. 30. 
organiques, à savoir les nécessités qui lui sont intérieures. Le roman «vit selon ses propres lois» écrit Malraux dans L'homme précaire et la littérature, c'est-à-dire conformément à «sa cohérence propre, aussi rigoureuse que celle à laquelle le monde musical subordonne ses livrets ou ses ballets» (HPL, p. 171).

Tous les romanciers-essayistes esquissent, à un moment ou à un autre, le croquis du corps "physique» du roman ou encore ciblent certaines parties de son anatomie: sa silhouette, son appétit, sa digestion, ses pathologies, ses mensurations, sa vitalité sexuelle, etc. Chez Félicien Marceau, ces références à un corps physique s'établissent dans un lien direct avec la question des lois: «Au bout de quelques pages, le roman prend corps, et ce corps a sa propre gymnastique, il commence à sécréter des lois qui ne sont plus tout à fait celles de l'auteur. C'est un combat, un roman. Dans un combat, on ne sait pas qui va périr et qui va survivre.» $(R L, \mathrm{p} .11)$

Marceau s'attarde plus spécifiquement à un élément du corps physiologique du roman: ses «sécrétions», soit les traces des lois biologiques qui gouvernent ce corps, provenant des combats qui ont lieu à l'échelle microscopique. Nous ne sommes qu'au tout début de l'essai, mais l'image est insistante et elle revient une centaine de pages plus loin:

$[\mathrm{T}]$ rès rapidement, le roman va imposer son mouvement, ses choix, sa rigueur. De tous ces éléments, le romancier n'en gardera peut-être aucun. Mais il n'en est aucun non plus qu'il puisse rejeter. Dans cet immense bric-à-brac qui l'entoure, tout peut servir. Le romancier va-t-il au moins trouver dans la forme même du roman de quoi le guider? Même pas. Ce roman va-t-il avoir cent cinquante pages ou trois milles? Au romancier à décider. [...] Aura-t-il des chapitres de cent pages comme Proust ou de sept lignes comme Morand? Au romancier de choisir. Va-t-il commencer par le commencement comme Stendhal dans La Chartreuse de Parme, par la fin comme François Mauriac dans Thérèse Desqueyroux ou par le milieu comme Balzac dans Le curé de Tours? Va-t-il brouiller la chronologie comme Aldous Huxley dans La paix des profondeurs ou l'observer avec la même rigueur que Sartre dans Les chemins de la liberté? Au romancier de voir. Là encore, le roman va imposer sa loi. Mais c'est une loi qu'il sécrète lui-même, qui n'a de sens que pour ce roman-là. C'est dire que cette loi n'existe pas. (RL, p. 111) 


\section{Comment le problème de la définition demeure entier}

Les romanciers-essayistes exaltent si bien la liberté du genre romanesque que plus on les lit, plus il devient paradoxalement difficile de dire ce qu'est un roman. Cette difficulté est exacerbée par le fait qu'ils parlent autant des possibilités du roman que du roman lui-même, comme en fait foi ce passage de Kundera: «[L|'histoire du roman a pris le chemin qu'elle a pris. Elle aurait pu en prendre un autre. La forme du roman est liberté quasi illimitée. Le roman durant son histoire n'en a pas beaucoup profité. Il a manqué cette liberté. Il a laissé beaucoup de possibilités formelles inexploitées ${ }^{17}$.»

Dans cette spirale aux ressorts tautologiques ne reste peut-être que l'image d'un "immense bric-à-brac», selon l'expression de Marceau évoquée plus haut, où tout cohabite avec tout, "où tout peut servir» $(R L, \mathrm{p} .111)$, ou encore celle de Milan Kundera pour qui la liberté du roman est celle d'un «superbe désordre» (TT, p. 29), deux images qui ne sont pas sans faire écho à la boutique hétéroclite du vieil antiquaire évoquée par Jacques Rancière. Plus la liberté est convoquée, plus il devient difficile de dire ce qu'est un genre narratif «libre». Mais les romanciers, comme le rappelle Félicien Marceau, ne sont pas dupes de la difficulté posée par le fait de chercher à définir le roman par sa liberté: «Mais quelle définition pourrait-on proposer pour un genre (et sous ce mot trop précis déjà le romancier se cabre), pour un genre, dis-je, qui s'est arrogé tant de libertés et qui probablement en prendra encore d'autres?» ( $R L$, p. 104)

C'est que, malgré les apparences, la «liberté» du roman n'est peut-être pas une affaire de définition. Elle est moins un fait (ou une donnée préalable) qu'un idéal à atteindre, une tâche qu'il incombe au romancier de réaliser. En outre, s'insurger contre les règles comme le fait Jacques Laurent n'est peut-être qu'une façon de se donner le sentiment de les transgresser, car pour enfreindre les lois du genre, il faut d'abord les poser comme existantes. C'est du moins vers cette piste que nous oriente l'essai d'Aragon, Comment j'ai appris à écrire ou Les incipit, qui porte davantage sur ses propres romans que sur le genre en soi. Aragon raconte ainsi le projet de son impossible roman, La défense de l'infini:

17. Milan Kundera, L'art du roman [1986], Paris, Gallimard, coll. «Folio Essais», 1995, p. 103. 
[J]e cherchais, en même temps que je poursuivais le défi d'un roman-comble, d'un «roman des romans» (j'avais songé à l'annoncer ainsi), à faire paraître à partir du roman reconnu comme tel, une nouvelle espèce de roman enfreignant toutes les lois traditionnelles de ce genre, qui ne soit ni un récit (une histoire) ni un personnage (un portrait), et que la critique devrait par suite envisager les mains nues, sans aucune arme avec lesquelles elle a beau jeu d'exercer sa stupide cruauté, puisqu'il n'y avait plus de règles du genre ${ }^{18}$.

Tout porte à croire que le romancier a besoin que des règles existent et si elles n'existent pas, il est prêt à les inventer précisément dans le but de les dépasser.

Enfin, insister autant sur la liberté du roman est certainement une façon pour un romancier-essayiste de se conférer un certain pouvoir, comme le font Malraux, lorsqu'il associe "ubiquité», «omniscience» et «liberté» (HPL, p. 111), Laurent quand il érige le romancier en « démiurge» ( $R R$, p. 63), ou encore Alain Robbe-Grillet dans Pour un nouveau roman lorsqu'il écrit: «Ce qui fait la force du romancier c'est justement qu'il invente, qu'il invente en toute liberté, sans modèle ${ }^{19}$.» Se proclamer libre et tout-puissant comme le font à peu près tous les romanciers-essayistes de la seconde moitié du $\mathrm{xx}^{\mathrm{e}}$ siècle, c'est aussi chercher à faire du roman une création, l'invention d'un monde, plutôt que son miroir.

18. Aragon, Comment j'ai appris à écrire ou Les incipit, Genève, Skira, 1969, p. 54.

19. Alain Robbe-Grillet, Pour un nouveau roman, Paris, Minuit, 1963, p. 30. 